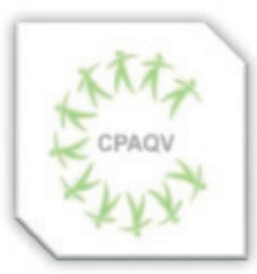

ISSN: $2178-7514$

Vol. $13\left|\mathbf{N}^{\circ} .2\right|$ Ano 2021
ARTIGO DE REVISÃO

\section{PREVALÊNCIA DE SOBREPESO E OBESIDADE INFANTIL E FATORES DE RISCOS CARDIOVASCULARES}

Prevalence of child overweight and obesity and cardiovascular risk factors

José Leonan Gomes Rodrigues ${ }^{1}$, Suellen da Silva Mendonça ${ }^{1}$, Daniel Castro Fernandes ${ }^{1}$, Ana Viviane Herculano Santiago Silva ${ }^{1}$, Antônio Gabriel Pantoja Silva Santos ${ }^{1}$, Thaise Maria Oliveira Maciel ${ }^{1}$, Luiz Otávio Franco Corrêa ${ }^{1}$, José Maria Dias Neto ${ }^{2}$, Rafael da Rocha Dias Quintairos ${ }^{2}$, Kethelenn Caroline Leão Lutitto², João Helder Castro dos Santos ${ }^{2}$, Amanda Jordana Silva Souza ${ }^{3}$, Tereza Cristina dos Reis Ferreira ${ }^{4}$

\begin{abstract}
RESUMO
Introdução: Segundo a Organização Mundial da Saúde (OMS), o sobrepeso e a obesidade infantil são um dos mais graves problemas de saúde e estimativas mostram que em 2025 poderá chegar a 75 milhões (SCARAFICCI et al, 2020). A partir disso, parâmetros são necessários para avaliar os riscos e os níveis de sobrepesos e obesidade nessas crianças e adolescentes. Objetivo: Analisar por meio da literatura como a obesidade interfere na saúde cardiovascular de crianças e adolescentes. Método: Trata-se de uma revisão narrativa com base em artigos publicados de 2011 a 2020 nas bases de dados SCIELO, BVS, LILACS, MEDLINE, PUB MED, PEDro, Cochrane e Google Acadêmico. Foram utilizados os seguintes descritores: Sobrepeso infantil; Obesidade infantil; Fatores de riscos; Riscos cardiovasculares. Resultado: Mudanças em relação à prática de exercício físico e alimentação relacionam-se com diminuição do índice de massa corporal, circunferência da cintura, massa gorda e triglicerídeos em grande parte dos artigos encontrados, além disso, os comportamentos relacionados com dietas e atividade física influenciam nos marcadores de saúde vascular de forma variável em crianças e adolescentes. Conclusão: É notório que a obesidade interfere de maneira concisa na saúde cardiovascular de crianças e adolescentes, ao modo que a obesidade predispõe ao desenvolvimento de algumas síndromes metabólicas como a diabetes e a dislipidemia.
\end{abstract}

Palavras-chave: Sobrepeso infantil; Obesidade infantil; Fatores de riscos; Riscos cardiovasculares.

\begin{abstract}
Introduction: According to the World Health Organization (WHO), overweight and childhood obesity are one of the most serious health problems and estimates show that in 2025 it may reach 75 million (SCARAFICCI et al, 2020). From this, parameters are needed to assess the risks and levels of overweight and obesity in these children and adolescents. Objective: To analyze through the literature how obesity interferes in the cardiovascular health of children and adolescents. Method: This is a narrative review based on articles published in 2011 to 2020 in the databases SCIELO, BVS, LILACS, MEDLINE, PUB MED, PEDro, Cochrane and Google Scholar. The following descriptors were used: Child overweight; Child obesity; Risk factors; Cardiovascular risks. Result: Changes in relation to the practice of physical exercise and diet are related to a decrease in body mass index, waist circumference, fat mass and triglycerides in most of the articles found, in addition, the behaviors related to diets and physical activity influence in vascular health markers in a variable way in children and adolescents. Conclusion: It is well known that obesity concisely interferes with the cardiovascular health of children and adolescents, as obesity predisposes to the development of some metabolic syndromes such as diabetes and dyslipidemia.
\end{abstract}

Keywords: Child overweight; Child obesity; Risk factors; cardiovascular risks

1- Discentes do Curso de Fisioterapia da Universidade do Estado do Pará (UEPA)

2- Discentes do Curso de Fisioterapia da Facukdade Paraense de Ensino (FAPEN)

3- Fisioterapeuta graduada pela da Universidade do Estado do Pará (UEPA)

4- Fisioterapeuta, Professora do Curso de Fisioterapia da Universidade do Estado Pará (UEPA). Dra Em Ciências da Reabilitação (UNINOVE)

Autor de correspondência

José Leonan Gomes Rodrigues - Email: jleonam02@gmail.com

DOI: $10.36692 / \mathrm{v} 13 \mathrm{n} 2-06 \mathrm{R}$ 


\section{INTRODUÇÃO}

A obesidade é definida como um excesso de gordura corporal, sendo uma doença que afeta todo organismo, desencadeado por hábitos alimentares não saudáveis, abrangendo aspectos clínicos, epidemiológicos e psicossociais, necessitando de uma abordagem multidisciplinar para seu controle ${ }^{1}$. A partir disso, parâmetros são necessários para avaliar os riscos e os níveis de sobrepesos e obesidade nessas crianças e adolescentes, sendo utilizado a antropometria (peso, estatura), o Índice de Massa Corporal (IMC), utilizando a curva da Organização Mundial de Saúde (OMS) de 2006, onde considera o percentil para sobrepeso e obesidade de 85 a $93 \%$, respectivamente ${ }^{2}$.

Segundo a OMS, o sobrepeso e a obesidade infantil são um dos mais graves problemas de saúde e estimativas mostram que em 2025 poderá chegar a 75 milhões 2 . Um estudo brasileiro, realizado em 73.399 escolares de 12 a 17 anos, para investigação de Estudo de Riscos Cardiovasculares em Adolescentes (ERICA), observou que 1 em cada 5 adolescentes poderiam não ser hipertensos se não fossem obesos ${ }^{3}$.

Nesse sentido, esse quadro de sobrepeso intensifica o desenvolvimento de diversas complicações cardiovasculares como hipertensão arterial e arteriosclerose além de outras enfermidades tais como diabetes, distúrbios psicológicos e disfunções articulares ${ }^{1}$. Ademais, as mudanças nos comportamentos dietéticos e na atividade física, como a ingestão de gordura e inatividade física, potencialmente contribuem a redução nos marcadores de saúde vascular, porém esses fatores ainda são variáveis. ${ }^{4}$

Diante do exposto, este estudo tem como objetivo verificar na literatura as publicações referentes a prevalência de sobrepeso e obesidade infantil e fatores de riscos cardiovasculares.

\section{MÉTODOS}

O presente estudo trata-se de uma revisão narrativa da literatura, que foi feita por meio de uma busca nas bases de dados Scielo, LILACS, MEDLINE/Pubmed, PEDro, Crochena e Google acadêmico, sendo contabilizados para a amostra materiais com data de publicação dos anos de 2011 a 2020.

Para a realização da busca dos artigos, foram usados os seguintes descritores como estratégia de busca: "Sobrepeso infantil; Obesidade infantil; Fatores de riscos; Riscos cardiovasculares". Usando os operadores booleanos and, or ou not.

A revisão dos artigos foi realizada em duas etapas. Inicialmente, os artigos encontrados nas bases de dados foram avaliados quanto ao título, leitura do resumo e palavras-chave, sendo eleitos os que mencionaram o atendimento e assistência as gestantes no sistema único de Saúde na atenção básica. 


\section{RESULTADOS}

Os artigos selecionados foram pesquisados nas plataformas BVS e Pubmed. Os termos buscados foram: Sobrepeso, obesidade infantil, fatores de risco, risco cardiovasculares e epidemiologia, em inglês foram utilizados os descritores: cardiovasculares risk, overweight, pediatric obesity, risk factors, epidemiology. Após as aplicações dos filtros referentes aos anos de 2011-2021 e as combinações dos termos com o operador AND, encontrou-se 42 artigos, depois da leitura dos textos completos dos artigos, foram selecionados apenas 13 artigos, incluindo estudos de prevalência, ensaio clinico, e ensaio clinico randomizado.

\section{QUADRI 1- ANEXADO}

\section{DISCUSSÃO}

De maneira geral, a obesidade na infância e adolescência é consequência de maus hábitos alimentares (fast-foods, transgênicos, alimento rico em sódio) e o sedentarismo. Isso deve ao fato de que, no contexto atual, tais indivíduos são expostos a esse estilo de vida prematuramente. Além disso, com o avanço tecnológico, o uso de equipamentos eletrônicos é mais comum e geralmente não incentiva a pratica de atividade física $^{5}$.

A somação de todos esses fatores aumenta o risco cardiovascular, tal como o desenvolvimento precoce de síndromes metabólicas, a exemplo: hipertensão arterial sistêmica, dislipidemia e diabetes mellitus6. Para analisar e avaliar os riscos de desenvolvimento de doenças cardiovasculares associadas a obesidade ou sobrepeso, pode se evidenciada por meio de escala de avaliação, sendo elas: IMC, Circunferência da cintura, pressão $\operatorname{arterial}^{78} 9$.

A dislipidemia que é o aumento de colesterol e triglicerídeos no sangue, durante a infância e adolescência, vem sendo objeto de diversos estudos, sobretudo pela grande incidência e por contribuir como um fator preditor da aterosclerose na fase adulta. Além disso, estudos indicam que a ocorrência de dislipidemia no período da infância e adolescência está associada com eventos cardiovasculares e com aptidão cardiorrespiratória (APCR). Da mesma forma, os aspectos comportamentais, envolvendo hábitos alimentares e comportamento sedentário, com redução do gasto calórico e da prática de atividade física, bem como maior tempo de uso de recursos eletrônicos têm sido introduzidos como importantes fatores que determinam o risco cardiovascular em crianças e adolescentes10.

Essas altas taxas da prevalência de obesidade na infância vêm preocupando os profissionais da área de saúde, por isso estão sendo feitas pesquisas a respeito da prevenção, causas e tratamentos. Discutir as consequências da obesidade em crianças e suas relações com a orientação dos pais têm impactos diretos na implantação de ações favoráveis, tanto no consultório médico quanto nos hábitos familiares. Sociedade, governo e famílias em conjunto, 
podem se beneficiar da discussão acerca da orientação dos pais na primeira consulta dos filhos e de seus impactos ${ }^{2}$.

\section{CONCLUSÃO}

Conclui-se de forma preponderante que a obesidade interfere de maneira concisa na saúde cardiovascular de crianças e adolescentes, ao modo que a obesidade predispõe ao desenvolvimento de algumas síndromes metabólicas como a diabetes e a dislipidemia.

Outrossim, na análise dos artigos do presente estudo de revisão, torna-se evidente que o estilo de vida e um meio de intervenção que pode modificar a influência da obesidade sobre o sistema cardiovascular de crianças e adolescentes, ao modo que a pratica de exercícios físicos associada a uma alimentação regrada pode se caracterizar como mecanismo de adequação da saúde cardiovascular e minimizar os fatores de riscos que predispõe ao desenvolvimento de outras síndromes metabólicas, já citadas anteriormente.

Nesse sentido, um estilo de vida mais saudável favorece efeitos positivos em crianças, tendo efeito na massa de gordura, na pressão arterial, nos níveis de insulina e na função cardiovascular geral, provocando também uma redução da circunferência da cintura abdominal, que também se configura como um meio de quantificar o risco para a saúde cardiovascular. Assim sendo, é importante também reduzir esse nível de obesidade na fase da infância e adolescência, haja vista que tendem a persistir com o envelhecimento, atrelada com a diminuição da expectativa de vida, além de proporcionar a disfunção epitelial, que atua também como um marcador em relação a complicações cardiovasculares.

É importante ressaltar a escassez de pesquisas relacionadas a essa classe, já que existe poucos artigos científicos que evidenciem a influência da obesidade em crianças e adolescentes.

\section{REFERÊNCIAS}

1 GARCIA, Amanda Vitória Rosa, PAIVA, Priscilla Moraes Henrique. Obesidade infantil no Brasil: Revisão Bibliográfica. FEPESMIG, 2020.

2- SCARAFICCI, Ana Cláudia et al. Obesidade infantil: recomendações para orientação inicial. CuidArte, Enferm, p. 257-263, 2020.

3- BLOCH, Katia Vergetti et al.: prevalências de hipertensão arterial e obesidade em adolescentes brasileiros. Revista de Saúde Pública, v. 50, p. 9s, 2016.

4- RYDER JR, Vega-López S, Gaesser GA, Buman MP, Shaibi GQ. Heterogeneous vascular responses to lifestyle intervention in obese Latino adolescents. Metab Syndr Relat Disord. 2014 Dec;12(10):509-15.

5- DE OLIVEIRA, Grasiela Junges et al. Comparação das curvas NCHS, CDC e OMS em crianças com risco cardiovascular. Revista da Associação Médica Brasileira, v. 59, n. 4, p. 375-380, 2013.

6- RYDER, Justin R. et al. Heterogeneous vascular responses to lifestyle intervention in obese latino adolescents. Metabolic syndrome and related disorders, v. 12 , n. 10 , p. 509-515, 2014.

7- ANDAKI, Alynne Christian Ribeiro et al. Waist circumference percentile curves as a screening tool to predict cardiovascular risk factors and metabolic syndrome risk in Brazilian children. Cadernos de saúde pública, v. 34, p. e00105317, 2018.

8- PAZIN, Daiane Cristina et al. Waist circumference is associated with blood pressure in children with normal body mass index: a cross-sectional analysis of 3,417 school children. Arquivos brasileiros de cardiologia, v. 109, n. 6, p. 509-515, 2017.

9- GRÖBER-GRÄTZ, Dagmar et al. Body mass index or waist circumference: which is the better predictor for hypertension and dyslipidemia in overweight/obese children and adolescents? Association of cardiovascular risk related to body mass index or waist circumference. Hormone research in paediatrics, v. 80, n. 3, p. 170-178, 2013. 
10- REUTER CP, Brand C, Silva PT, Reuter EM, Renner JDP, Frank SIR et al. Relationship between Dyslipidemia, Cultural Factors, and Cardiorespiratory Fitness in Schoolchildren. Arquivo Brasileiro de Cardiologia. 2019; (in press).

11- PARILLO, M. et al. Metabolic changes after a hypocaloric, low-glycemic-index diet in obese children. Journal of endocrinological investigation, v. 35, n. 7, p. 629-633, 2012.

PACIFICO, L. et al. Arterial function and structure after a 1-year lifestyle intervention in children with nonalcoholic fatty liver disease. Nutrition, Metabolism and Cardiovascular Diseases, v. 23, n. 10, p. 1010-1016, 2013.

12- REUTER, Cezane Priscila et al. Prevalência de obesidade e risco cardiovascular em crianças e adolescentes do município de Santa Cruz do Sul, Rio Grande do Sul. São Paulo Medical Journal, v. 131, n. 5, p. 323-330, 2013.

13- MARCUS, Marsha D.; FOSTER, Gary D.; EL GHORMLI, Laure. Estabilidade da categoria de peso relativo e fatores de risco cardiometabólico entre jovens do ensino médio com obesidade moderada e severa. Obesidade, v. 22, n. 4, pág. 1118-1125, 2014.

14- MÜLLER, Ulrike M. et al. Long term impact of one daily unit of physical exercise at school on cardiovascular risk factors in school children. European journal of preventive cardiology, v. 23, n. 13, p. 1444-1452, 2016.

15- FALBE, Jennifer et al. Famílias ativas e saudáveis: um ensaio clínico randomizado de uma intervenção de obesidade adaptada culturalmente para crianças latinas. Pediatria acadêmica, v. 15, n. 4, pág. 386-395, 2015.

16- ROSINI, Nilton et al. Metabolic syndrome and importance of associated variables in children and adolescents in Guabiruba-SC, Brazil. Arquivos brasileiros de cardiologia, v. 105, n. 1, p. 37-44, 2015.

17- ANDAKI, Alynne Christian Ribeiro et al. Waist circumference percentile curves as a screening tool to predict cardiovascular risk factors and metabolic syndrome risk in Brazilian children. Cadernos de saúde pública, v. 34, p. e00105317, 2018.

18- PENHA, Jociene Terra da et al. Physical fitness and activity, metabolic profile, adipokines and endothelial function in children. J. Pediatr. (Rio J.) Porto Alegre,v. 95, n. 5, p. 531-537, Sept. 2019.

\section{OBSERVAÇÃO: Os autores declaram não existir}

conflitos de interesse de qualquer natureza. 
QUADRO 1- ARTIGOS PUBLUCIDADOS DE 2011 A 2012

\begin{tabular}{|c|c|c|c|c|c|c|}
\hline Título & $\begin{array}{c}\text { Ano de } \\
\text { publicação }\end{array}$ & Autores & $\begin{array}{l}\text { Tipo de } \\
\text { estudo }\end{array}$ & Metodologia & Resultados & Conclusão \\
\hline $\begin{array}{l}\text { Metabol } \\
\text { ic } \\
\text { change } \\
\text { s after a } \\
\text { hypocal } \\
\text { oric, } \\
\text { low- } \\
\text { glycemi } \\
\text { c-index } \\
\text { diet in } \\
\text { obese } \\
\text { children }\end{array}$ & 2012 & $\begin{array}{l}\text { M. Parillo, M. } \\
\text { R. Licenziati, } \\
\text { M. Vacca, D. } \\
\text { De Marco \& } \\
\text { A. lannuzzi } \\
\text { MD }\end{array}$ & $\begin{array}{l}\text { Ensaio } \\
\text { clínico } \\
\text { controlado } \\
\text { randomiza } \\
\text { do }\end{array}$ & $\begin{array}{l}\text { Vinte e seis } \\
\text { crianças com } \\
\text { sobrepeso ou } \\
\text { obesidade } \\
\text { foram divididas } \\
\text { em dois grupos } \\
\text { e submetidas a } \\
\text { diferentes } \\
\text { dietas, de baixo } \\
\text { e alto índice } \\
\text { glicêmico. }\end{array}$ & $\begin{array}{l}\text { Comparando } \\
\text { as dietas de } \\
\text { alto e baixo } \\
\text { índice } \\
\text { glicêmico, } \\
\text { ambas } \\
\text { promoveram } \\
\text { diminuição do } \\
\text { IMC, na } \\
\text { pressão } \\
\text { arterial e na } \\
\text { proteína C } \\
\text { reativa de alta } \\
\text { sensibilidade, } \\
\text { mas apenas } \\
\text { as dietas de } \\
\text { baixo índice } \\
\text { glicêmico } \\
\text { diminuíram a } \\
\text { circunferência } \\
\text { da cintura. }\end{array}$ & $\begin{array}{l}\text { A dieta hipocalórica de } \\
\text { baixo índice glicêmico } \\
\text { proporciona mais } \\
\text { efeitos benéficos do } \\
\text { que a dieta } \\
\text { hipocalórica de alto } \\
\text { índice glicêmico em } \\
\text { crianças obesas. }\end{array}$ \\
\hline $\begin{array}{l}\text { Arterial } \\
\text { function } \\
\text { and } \\
\text { structur } \\
\text { e after a } \\
\text { 1-year } \\
\text { lifestyle } \\
\text { interven } \\
\text { tion in } \\
\text { children } \\
\text { with } \\
\text { nonalco } \\
\text { holic } \\
\text { fatty } \\
\text { liver } \\
\text { disease }\end{array}$ & 2013 & $\begin{array}{l}\text { L Pacifico, } \\
\text { Arca, C } \\
\text { Anania, V } \\
\text { Cantisani, } \\
\text { Di Martino, } \\
\text { Chiesa }\end{array}$ & $\begin{array}{l}\text { Estudo } \\
\text { populacion } \\
\text { al } \\
\text { longitudina } \\
\text { I }\end{array}$ & $\begin{array}{l}\text { O estudo avaliou } \\
135 \quad \text { crianças } \\
\text { obesas, antes e } \\
\text { após a } \\
\text { intervenção com } \\
\text { educação } \\
\text { nutricional, dieta } \\
\text { hipocalórica e } \\
\text { exercício físico } \\
\text { por } 12 \text { meses. }\end{array}$ & $\begin{array}{l}\text { Mudanças em } \\
\text { relação à } \\
\text { prática de } \\
\text { exercício } \\
\text { físico } \\
\text { alimentação } \\
\text { relacionam-se } \\
\text { com } \\
\text { diminuição do } \\
\text { índice de } \\
\text { massa } \\
\text { corporal, } \\
\text { circunferência } \\
\text { da cintura, } \\
\text { massa gorda e } \\
\text { triglicerídeos. }\end{array}$ & $\begin{array}{l}\text { A intervenção no estilo } \\
\text { de vida é eficaz para a } \\
\text { redução dos fatores de } \\
\text { risco cardiovascular }\end{array}$ \\
\hline
\end{tabular}




\begin{tabular}{|c|c|c|c|c|c|c|}
\hline $\begin{array}{l}\text { Body } \\
\text { Mass } \\
\text { Index or } \\
\text { Waist } \\
\text { Circumf } \\
\text { erence: } \\
\text { Which } \\
\text { Is the } \\
\text { Better } \\
\text { Predicto } \\
\text { r for } \\
\text { Hyperte } \\
\text { nsion } \\
\text { and } \\
\text { Dyslipid } \\
\text { emia in } \\
\text { Overwei } \\
\text { ght/Obe } \\
\text { se } \\
\text { Childre } \\
n \text { and } \\
\text { Adolesc } \\
\text { ents? } \\
\text { Associa } \\
\text { tion of } \\
\text { Cardiov } \\
\text { ascular } \\
\text { Risk } \\
\text { Related } \\
\text { to Body } \\
\text { Mass } \\
\text { Index or } \\
\text { Waist } \\
\text { Circumf } \\
\text { erence }\end{array}$ & 2013 & $\begin{array}{l}\text { Gröber- } \\
\text { Grätz D. } \\
\text { Widhalm K. } \\
\text { Zwaan M. } \\
\text { Reinehr T. } \\
\text { Blüher S. } \\
\text { Schwab K.O. } \\
\text { Wiegand S. } \\
\text { Holl R.W. }\end{array}$ & $\begin{array}{l}\text { Ensaio } \\
\text { clínico }\end{array}$ & $\begin{array}{l}\text { Foi avaliado o } \\
\text { índice de massa } \\
\text { corporal, a } \\
\text { circunferência } \\
\text { da cintura, a } \\
\text { pressão } \\
\text { sanguínea e } \\
\text { lipídios de } 5.978 \\
\text { adolescentes de } \\
11 \text { a } 17,9 \text { anos } \\
\text { de idades. }\end{array}$ & & $\begin{array}{l}\text { O IMC está mais } \\
\text { associado } \\
\text { hipertensão, enquanto } \\
\text { a circunferência } \\
\text { cintura está mais } \\
\text { associada } \\
\text { dislipidemia. à } \\
\text { entanto, a medição } \\
\text { adicional da CC tem } \\
\text { apenas um pequeno } \\
\text { benefício em jovens } \\
\text { obesos. }\end{array}$ \\
\hline $\begin{array}{l}\text { Compar } \\
\text { ação } \\
\text { das } \\
\text { curvas } \\
\text { NCHS, } \\
\text { CDC e } \\
\text { OMS } \\
\text { em } \\
\text { criança } \\
\text { s com } \\
\text { risco } \\
\text { cardiov } \\
\text { ascular }\end{array}$ & 2013 & $\begin{array}{l}\text { Grasiela } \\
\text { Junges de } \\
\text { Oliveira, } \\
\text { Sandra Mari } \\
\text { Barbiero, } \\
\text { Claudia } \\
\text { Ciceri Cesa, } \\
\text { Lucia } \\
\text { Campos } \\
\text { Pellanda }\end{array}$ & $\begin{array}{l}\text { Estudo de } \\
\text { coorte }\end{array}$ & $\begin{array}{l}\text { O estudo incluiu } \\
118 \text { crianças e } \\
\text { adolescentes, } \\
\text { entre } 2 \text { e } 19 \\
\text { anos, } \text { que } \\
\text { possuíam } \\
\text { fatores de risco } \\
\text { cardiovasculare } \\
\text { s. }\end{array}$ & $\begin{array}{l}\text { A população } \\
\text { analisada é } \\
\text { em maioria } \\
\text { obesa, com a } \\
\text { estatura } \\
\text { adequada de } \\
\text { acordo com a } \\
\text { idade. }\end{array}$ & $\begin{array}{lr}\text { Observa-se } & \text { um } \\
\text { aumento da obesidade } \\
\text { e uma redução do } \\
\text { sobrepeso e } \\
\text { eutrofia. }\end{array}$ \\
\hline
\end{tabular}




\begin{tabular}{|c|c|c|c|c|c|c|}
\hline $\begin{array}{l}\text { Prevale } \\
\text { nce of } \\
\text { obesity } \\
\text { and } \\
\text { cardiov } \\
\text { ascular } \\
\text { risk } \\
\text { among } \\
\text { children } \\
\text { and } \\
\text { adolesc } \\
\text { ents in } \\
\text { the } \\
\text { municip } \\
\text { ality of } \\
\text { Santa } \\
\text { Cruz do } \\
\text { Sul, Rio } \\
\text { Grande } \\
\text { do Sul }\end{array}$ & 2013 & $\begin{array}{l}\text { Cézane } \\
\text { Priscila } \\
\text { Reuter, } \\
\text { Leandro } \\
\text { Tibiriçá } \\
\text { Burgos, } \\
\text { Marcelo Dias } \\
\text { Camargo, } \\
\text { Lia } \\
\text { Gonçalves } \\
\text { Possuelo, } \\
\text { Miriam } \\
\text { Beatris } \\
\text { Reckziegel, } \\
\text { Éboni Marília } \\
\text { Reuter, } \\
\text { Francielle } \\
\text { Pasqualotti } \\
\text { Meinhardt, } \\
\text { Miria Suzana } \\
\text { Burgos }\end{array}$ & $\begin{array}{l}\text { Estudo } \\
\text { transversal }\end{array}$ & $\begin{array}{l}\text { Esse estudo } \\
\text { transversal } \\
\text { inclui } 564 \\
\text { crianças re } \\
\text { adolescentes, } \\
\text { selecionados de } \\
\text { forma aleatória. }\end{array}$ & $\begin{array}{l}\text { Comparando } \\
\text { um grupo de } \\
\text { meninas e } \\
\text { meninos, as } \\
\text { meninas } \\
\text { apresentaram } \\
\text { maior } \\
\text { aumento do } \\
\text { percentual de } \\
\text { triglicerídeos } \\
\text { do que } \\
\text { meninos. } \\
\text { Analisando } \\
\text { outras } \\
\text { variáveis, os } \\
\text { indivíduos } \\
\text { com } \\
\text { obesidade e } \\
\text { sobrepeso } \\
\text { apresentaram } \\
\text { aumento da } \\
\text { circunferência } \\
\text { abdominal, } \\
\text { triglicerídeos e } \\
\text { pressão } \\
\text { arterial } \\
\text { sistólica. }\end{array}$ & $\begin{array}{l}\text { A prevenção de } \\
\text { sobrepeso } \\
\text { obesidade é } \\
\text { importante para } \\
\text { impedir a persistência } \\
\text { da situação e o agravo } \\
\text { ou surgimento de } \\
\text { doenças e hábitos não } \\
\text { saudáveis. }\end{array}$ \\
\hline $\begin{array}{l}\text { Heterog } \\
\text { eneous } \\
\text { vascula } \\
r \\
\text { respons } \\
\text { es to } \\
\text { lifestyle } \\
\text { interven } \\
\text { tion in } \\
\text { obese } \\
\text { Latino } \\
\text { adolesc } \\
\text { ents }\end{array}$ & 2014 & 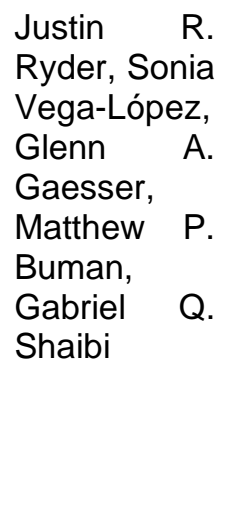 & & $\begin{array}{l}\text { Estudo com } \\
\text { quinze } \\
\text { adolescentes } \\
\text { com obesidade, } \\
\text { selecionados } \\
\text { para participar } \\
\text { de intervenções } \\
\text { no estilo de vida, } \\
\text { especificamente } \\
\text { em relação à } \\
\text { alimentação e } \\
\text { atividade física. }\end{array}$ & $\begin{array}{l}\text { Os } \\
\text { comportament } \\
\text { os } \\
\text { relacionados } \\
\text { com dietas e } \\
\text { atividade } \\
\text { física } \\
\text { influenciam } \\
\text { nos marcadores } \\
\text { de saúde } \\
\text { vascular de } \\
\text { forma variável. }\end{array}$ & $\begin{array}{l}\text { As mudanças no estilo } \\
\text { de vida dos jovens } \\
\text { proporcionam } \\
\text { benefícios em relação } \\
\text { à saúde vascular, } \\
\text { porém } \\
\text { respostas essas } \\
\text { heterogêneas. São }\end{array}$ \\
\hline
\end{tabular}




\begin{tabular}{|c|c|c|c|c|c|c|}
\hline $\begin{array}{l}\text { Stability } \\
\text { of } \\
\text { relative } \\
\text { weight } \\
\text { categor } \\
\text { y and } \\
\text { cardiom } \\
\text { etabolic } \\
\text { risk } \\
\text { factors } \\
\text { among } \\
\text { moderat } \\
\text { ely and } \\
\text { severel } \\
\text { y obese } \\
\text { middle } \\
\text { school } \\
\text { youth }\end{array}$ & 2014 & $\begin{array}{ll}\text { Marsha } & \text { D } \\
\text { Marcus, } & \\
\text { Gary D } & \\
\text { Foster, } & \\
\text { Laure } & \text { El } \\
\text { Ghormli } & \end{array}$ & $\begin{array}{l}\text { Ensaio } \\
\text { clínico } \\
\text { controlado } \\
\text { randomiza } \\
\text { do. }\end{array}$ & $\begin{array}{l}\text { Estudo } \\
\text { realizado com } \\
\text { crianças da } 6^{\underline{a}} \text { a } \\
8^{\underline{a}} \text { série, de } \\
\text { escolas } \\
\text { participantes do } \\
\text { programa } \\
\text { HEALTHY. As } \\
\text { avaliações } \\
\text { foram } \\
\text { padronizadas, } \\
\text { medindo altura, } \\
\text { peso, glicose, } \\
\text { insulina, lipídios } \\
\text { e pressão } \\
\text { arterial. }\end{array}$ & $\begin{array}{l}\text { Durante o } \\
\text { ensino médio, } \\
\text { a obesidade } \\
\text { grave teve } \\
\text { maior } \\
\text { permanência } \\
\text { do que a } \\
\text { obesidade } \\
\text { moderada, e } \\
\text { está } \\
\text { associada a } \\
\text { maiores níveis } \\
\text { de risco } \\
\text { cardiometabóli } \\
\text { co. }\end{array}$ & $\begin{array}{l}\text { Dados obtidos indicam } \\
\text { que a obesidade } \\
\text { pediátrica grave está } \\
\text { associada a um perfil } \\
\text { de risco } \\
\text { cardiometabólico } \\
\text { menor em relação à } \\
\text { obesidade moderada, } \\
\text { mas faltam dados } \\
\text { longitudinais de } \\
\text { amostras } \\
\text { comunidade. }\end{array}$ \\
\hline $\begin{array}{l}\text { Active } \\
\text { and } \\
\text { Healthy } \\
\text { Familie } \\
\text { s: A } \\
\text { Rando } \\
\text { mized } \\
\text { Controll } \\
\text { ed Trial } \\
\text { of a } \\
\text { Culturall } \\
\text { y } \\
\text { Tailored } \\
\text { Obesity } \\
\text { Interven } \\
\text { tion for } \\
\text { Latino } \\
\text { Childre } \\
n\end{array}$ & 2015 & $\begin{array}{l}\text { Jennifer } \\
\text { Falbe, } \\
\text { Annabelle A } \\
\text { Cadiz, } \\
\text { Nicole } \\
\text { Tantoco, } \\
\text { Hannah R } \\
\text { Thompson, } \\
\text { Kristine A } \\
\text { Madsen }\end{array}$ & $\begin{array}{l}\text { Ensaio } \\
\text { clínico } \\
\text { controlado } \\
\text { randomiza } \\
\text { do. }\end{array}$ & $\begin{array}{l}\text { Esse estudo foi } \\
\text { feito com } 55 \\
\text { grupos de pais e } \\
\text { filhos latinos, } \\
\text { com crianças de } \\
5 \text { a } 11 \text { anos, } \\
\text { participantes de } \\
\text { uma intervenção } \\
\text { multidisciplinar } \\
\text { de baixo custo. }\end{array}$ & $\begin{array}{l}\text { O IMC infantil } \\
\text { diminuiu (- } \\
0,50) \text { no grupo } \\
\text { de intervenção } \\
\text { e aumentou } \\
(+0,32) \quad \text { no } \\
\text { grupo de } \\
\text { controle. As } \\
\text { crianças } \\
\text { atribuídas à } \\
\text { intervenção } \\
\text { também } \\
\text { exibiram } \\
\text { melhorias } \\
\text { relativas sobre } \\
\text { os controles } \\
\text { no escore de } \\
\text { IMC } \\
\text { triglicerídeos. }\end{array}$ & $\begin{array}{l}\text { A intervenção } \\
\text { resultou em reduções } \\
\text { no IMC infantil, no } \\
\text { escore z do IMC e nos } \\
\text { triglicerídeos. } \\
\text { tratamento, que foi } \\
\text { projetado para famílias } \\
\text { latinas de baixa renda, } \\
\text { tem potencial para } \\
\text { reduzir } \\
\text { disparidades } \\
\text { saúde, mas estudos } \\
\text { futuros } \\
\text { necessários são } \\
\text { determinar o impacto a } \\
\text { longo prazo. }\end{array}$ \\
\hline $\begin{array}{l}\text { Metabol } \\
\text { ic } \\
\text { Syndro } \\
\text { me and } \\
\text { Importa } \\
\text { nce of } \\
\text { Associa } \\
\text { ted } \\
\text { Variable } \\
\mathrm{s} \text { in } \\
\text { Childre } \\
\mathrm{n} \text { and } \\
\text { Adolesc } \\
\text { ents in } \\
\text { Guabiru } \\
\text { ba - SC, } \\
\text { Brazil }\end{array}$ & 2015 & $\begin{array}{l}\text { Rosini, } \\
\text { Nilton; } \\
\text { Moura, } \\
\text { Solange A. } \\
\text { Z. } \\
\text { Oppermann; } \\
\text { Rosini, } \\
\text { Rodrigo } \\
\text { Diegoli; } \\
\text { Machado, } \\
\text { Marcos } \\
\text { José; Silva, } \\
\text { Edson Luiz } \\
\text { da. }\end{array}$ & $\begin{array}{l}\text { Estudo de } \\
\text { prevalênci } \\
\text { a. }\end{array}$ & $\begin{array}{l}\text { Estudo } \\
\text { realizado com } \\
1011 \\
\text { estudantes, } \\
\text { utilizando } \\
\text { amostras de } \\
\text { sangue } \\
\text { coletadas para } \\
\text { as medidas de } \\
\text { parâmetros } \\
\text { bioquímicos por } \\
\text { métodos } \\
\text { laboratoriais de } \\
\text { rotina. A } \\
\text { resistência à } \\
\text { insulina foi } \\
\text { estabelecida } \\
\text { pelo índice } \\
\text { HOMA-IR e } \\
\text { foram aferidos o }\end{array}$ & $\begin{array}{l}\text { A resistência à } \\
\text { insulina foi } \\
\text { mais } \\
\text { frequente nos } \\
\text { estudantes } \\
\text { com } \\
\text { sobrepeso } \\
(48 \%) \\
\text { obesos (41\%) } \\
\text { em } \\
\text { comparação } \\
\text { aos indivíduos } \\
\text { eutróficos. As } \\
\text { variáveis com } \\
\text { maior } \\
\text { influência para } \\
\text { o } \\
\text { desenvolvime } \\
\text { nto da SM } \\
\text { foram a }\end{array}$ & $\begin{array}{lr}\text { Foi observada elevada } \\
\text { prevalência de } & \text { SM nas } \\
\text { crianças } & \text { e } \\
\text { adolescentes } & \\
\text { avaliados. Estudantes } \\
\text { obesos, } & \text { com } \\
\text { sobrepeso } & \text { ou } \\
\text { resistentes à insulina } \\
\text { tiveram } & \text { maiores } \\
\text { chances } & \text { de } \\
\text { desenvolver } & \text { a } \\
\text { síndrome. } & \end{array}$ \\
\hline
\end{tabular}




\begin{tabular}{|c|c|c|c|c|c|c|}
\hline & & & & $\begin{array}{l}\text { peso, a altura, a } \\
\text { circunferência } \\
\text { da cintura e a } \\
\text { pressão arterial. }\end{array}$ & $\begin{array}{l}\text { obesidade, } \\
\text { sobrepeso, a } \\
\text { Rl e a idade. }\end{array}$ & \\
\hline $\begin{array}{l}\text { Long } \\
\text { term } \\
\text { impact } \\
\text { of one } \\
\text { daily } \\
\text { unit of } \\
\text { physical } \\
\text { exercis } \\
\text { e at } \\
\text { school } \\
\text { on } \\
\text { cardiov } \\
\text { ascular } \\
\text { risk } \\
\text { factors } \\
\text { in } \\
\text { school } \\
\text { children }\end{array}$ & 2016 & $\begin{array}{l}\text { Ulrike M M } \\
\text { Müller, } \\
\text { Claudia } \\
\text { Walther, } \\
\text { Volker } \\
\text { Adams, } \\
\text { Meinhard } \\
\text { Mende, } \\
\text { Jennifer } \\
\text { Adam, Kati } \\
\text { Fikenzer, } \\
\text { Katharina C } \\
\text { Machalica, } \\
\text { Sandra Erbs, } \\
\text { Axel Linke, } \\
\text { Gerhard } \\
\text { Schuler }\end{array}$ & $\begin{array}{l}\text { Ensaio } \\
\text { clínico } \\
\text { controlado } \\
\text { randomiza } \\
\text { do. }\end{array}$ & $\begin{array}{l}\text { Esse estudo foi } \\
\text { composto por } \\
366 \text { alunos do } 5^{\mathrm{a}} \\
\text { e } 6^{\mathrm{a}} \text { série, onde } \\
\text { o grupo de } \\
\text { intervenção } \\
\text { tinha aulas } \\
\text { extras } \\
\text { referentes a } \\
\text { condicionament } \\
\text { o físico e os } \\
\text { fatores de risco } \\
\text { cardiovascular. }\end{array}$ & $\begin{array}{l}\text { O consumo de } \\
\text { oxigênio de } \\
\text { pico foi } \\
\text { significativam } \\
\text { ente melhor } \\
\text { no grupo de } \\
\text { intervenção no } \\
\text { primeiro e no } \\
\text { segundo ano } \\
\text { de } \\
\text { acompanham } \\
\text { ento. Após } 4 \\
\text { anos, não } \\
\text { encontramos } \\
\text { mais nenhuma } \\
\text { diferença no } \\
\text { condicioname } \\
\text { nto físico. Os } \\
\text { alunos no } \\
\text { grupo de } \\
\text { intervenção } \\
\text { eram mais } \\
\text { propensos a } \\
\text { ter percentis } \\
\text { de índice de } \\
\text { massa } \\
\text { corporal } \\
\text { saudável em } \\
\text { comparação } \\
\text { com o grupo } \\
\text { de controle. }\end{array}$ & $\begin{array}{l}\text { Durante um período } \\
\text { de } 1-2 \text { anos, aulas } \\
\text { adicionais } \\
\text { exercícios físicos na } \\
\text { escola resultaram em } \\
\text { uma melhora do } \\
\text { condicionamento } \\
\text { físico. No entanto, o } \\
\text { acompanhamento de } \\
\text { longo prazo não } \\
\text { conseguiu demonstrar } \\
\text { a melhoria contínua do } \\
\text { desempenho na } \\
\text { intervenção em } \\
\text { comparação com o } \\
\text { grupo de controle. No } \\
\text { entanto, o grupo de } \\
\text { intervenção } \\
\text { apresentou taxas mais } \\
\text { baixas de índice de } \\
\text { massa corporal acima } \\
\text { do percentil } 90 \text { ao } \\
\text { longo de todo o } \\
\text { acompanhamento. }\end{array}$ \\
\hline $\begin{array}{l}\text { Waist } \\
\text { Circumf } \\
\text { erence } \\
\text { is } \\
\text { Associa } \\
\text { ted with } \\
\text { Blood } \\
\text { Pressur } \\
\text { e in } \\
\text { Childre } \\
\text { n with } \\
\text { Normal } \\
\text { Body } \\
\text { Mass } \\
\text { Index: A } \\
\text { Cross- } \\
\text { Section } \\
\text { al } \\
\text { Analysi } \\
\text { s of } 3 \text {, } \\
417 \\
\text { School }\end{array}$ & 2017 & $\begin{array}{l}\text { Pazin, } \\
\text { Daiane } \\
\text { Cristina; } \\
\text { Rosaneli, } \\
\text { Caroline } \\
\text { Filla; } \\
\text { Olandoski, } \\
\text { Márcia; } \\
\text { Oliveira, } \\
\text { Edna Regina } \\
\text { Netto de; } \\
\text { Baena, } \\
\text { Cristina } \\
\text { Pellegrino; } \\
\text { Figueredo, } \\
\text { Alyne S; } \\
\text { Baraniuk, } \\
\text { Analin Ono; } \\
\text { Kaestner, } \\
\text { Tatiana } \\
\text { Lorena da } \\
\text { Luz; Guarita- }\end{array}$ & $\begin{array}{l}\text { Estudo de } \\
\text { prevalênci } \\
\text { a. }\end{array}$ & $\begin{array}{l}\text { Estudo } \\
\text { realizado com } \\
\text { estudantes de } \\
\text { idades entre } 6 \text { e } \\
11 \text { anos com } \\
\text { índice de massa } \\
\text { corporal normal. } \\
\text { A circunferência } \\
\text { da cintura foi } \\
\text { categorizada } \\
\text { por quartil para } \\
\text { cada raixa } \\
\text { etária. } \\
\text { pressão arterial } \\
\text { normal } \\
\text { definida como } \\
\text { valores } \\
\text { percentil } \\
\text { Níveis } \\
\text { dessa } \\
\text { foram }\end{array}$ & 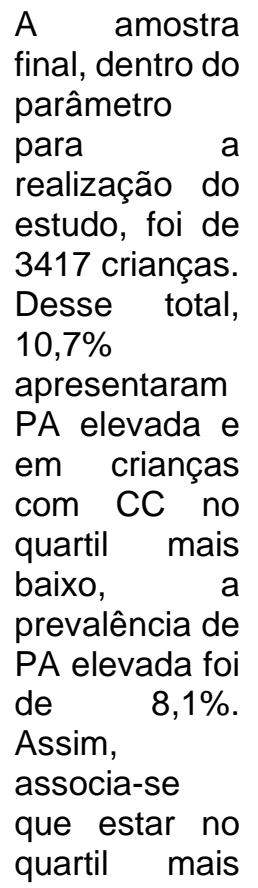 & $\begin{array}{l}\text { A circunferência da } \\
\text { cintura mostrou ser } \\
\text { forte preditor de } \\
\text { fatores de risco } \\
\text { cardiovasculares e } \\
\text { síndrome metabólica } \\
\text { em crianças. }\end{array}$ \\
\hline
\end{tabular}




\begin{tabular}{|c|c|c|c|c|c|c|}
\hline $\begin{array}{l}\text { Childre } \\
\mathrm{n}\end{array}$ & & $\begin{array}{l}\text { Souza, Luiz } \\
\text { Cesar; Faria- } \\
\text { Neto, José } \\
\text { Rocha. }\end{array}$ & & $\begin{array}{l}\text { considerados } \\
\text { elevados. }\end{array}$ & 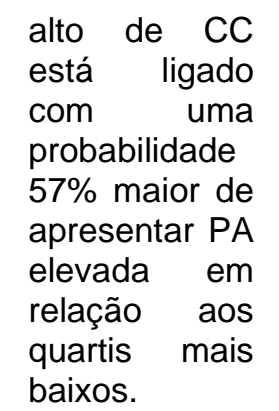 & \\
\hline $\begin{array}{l}\text { Waist } \\
\text { circumf } \\
\text { erence } \\
\text { percenti } \\
\text { le } \\
\text { curves } \\
\text { as a } \\
\text { screeni } \\
\text { ng tool } \\
\text { to } \\
\text { predict } \\
\text { cardiov } \\
\text { ascular } \\
\text { risk } \\
\text { factors } \\
\text { and } \\
\text { metabol } \\
\text { ic } \\
\text { syndro } \\
\text { me risk } \\
\text { in } \\
\text { Brazilia } \\
n \\
\text { children }\end{array}$ & 2018 & $\begin{array}{l}\text { Andaki, } \\
\text { Alynne } \\
\text { Christian } \\
\text { Ribeiro; } \\
\text { Mendes, } \\
\text { Edmar } \\
\text { Lacerda; } \\
\text { Santos, } \\
\text { Amanda; } \\
\text { Brito, Ciro } \\
\text { Jose; } \\
\text { Tinôco, } \\
\text { Adelson Luiz } \\
\text { Araújo; } \\
\text { Mota, Jorge. }\end{array}$ & $\begin{array}{l}\text { Estudo de } \\
\text { prevalênci } \\
\text { a. }\end{array}$ & $\begin{array}{l}\text { Incluiu } 1.397 \\
\text { crianças entre } 6 \\
\text { e } 10 \text { anos de } \\
\text { idade, através } \\
\text { de amostragem } \\
\text { probabilística } \\
\text { em escolas } \\
\text { públicas e } \\
\text { privadas em } \\
\text { Uberaba, Minas } \\
\text { Gerais, Brasil. } \\
\text { Foram } \\
\text { coletados dados } \\
\text { da perimetria da } \\
\text { cintura (PC), da } \\
\text { amostra de } \\
\text { sangue } \\
\text { medidas de } \\
\text { pressão arterial } \\
\text { para determinar } \\
\text { o diagnóstico de } \\
\text { síndrome } \\
\text { metabólica. }\end{array}$ & $\begin{array}{l}\text { Da amostra total, } \\
11,3 \% \\
\text { apresentaram } \\
\text { hipertensão } \\
\text { arterial, 0,9\% } \\
\text { apresentaram } \\
\text { hiperglicemia, } \\
43,1 \% \text { com HDL- } \\
\text { c abaixo do } \\
\text { recomendado e } \\
12,2 \% \text { com } \\
\text { hipertrigliceride } \\
\text { mia. } \\
\text { prevalência de } \\
\text { sobrepeso foi de } \\
13,5 \% \text {, } \\
\text { obesidade com } \\
5,0 \% \text { e SM com } \\
3,5 \% \text { em relação } \\
\text { ao total da } \\
\text { amostra. }\end{array}$ & $\begin{array}{l}\text { Por meio desse } \\
\text { estudo, conclui-se que } \\
\text { a circunferência da } \\
\text { cintura é um grande } \\
\text { preditor de fatores de } \\
\text { risco cardiovasculares } \\
\text { em crianças. }\end{array}$ \\
\hline $\begin{array}{l}\text { Physica } \\
\text { I fitness } \\
\text { and } \\
\text { activity, } \\
\text { metabol } \\
\text { ic } \\
\text { profile, } \\
\text { adipoki } \\
\text { nes and } \\
\text { endothe } \\
\text { lial } \\
\text { function } \\
\text { in } \\
\text { children }\end{array}$ & 2019 & $\begin{array}{l}\text { Jociene } \\
\text { Terra da } \\
\text { Penha, } \\
\text { Fernanda } \\
\text { Mussi } \\
\text { Gazolla, } \\
\text { Cecília } \\
\text { Noronha de } \\
\text { Miranda } \\
\text { Carvalho, } \\
\text { Isabel Rey } \\
\text { Madeira, } \\
\text { Flávio } \\
\text { Rodrigues- } \\
\text { Júnior, } \\
\text { Elisabeth de } \\
\text { Amorim } \\
\text { Machado, } \\
\text { Fernando } \\
\text { Lencastre } \\
\text { Sicuro, } \\
\text { Paulo }\end{array}$ & $\begin{array}{l}\text { Estudo } \\
\text { transversal }\end{array}$ & $\begin{array}{l}\text { Esse estudo } \\
\text { envolveu } \\
\text { crianças de 5-12 } \\
\text { anos. Sendo } \\
\text { avaliado pela } \\
\text { ipletismografia } \\
\text { de oclusão } \\
\text { venosa, os } \\
\text { níveis séricos de } \\
\text { adiponectina, } \\
\text { leptina, insulina } \\
\text { e lipidograma, } \\
\text { escore de } \\
\text { atividade física } \\
\text { (questionário } \\
\text { PAQ-C) e e } \\
\text { avaliação da } \\
\text { aptidão física } \\
\text { (teste Yo-yo). }\end{array}$ & $\begin{array}{l}\text { Um total de } 62 \\
\text { crianças } \\
\text { participou } \\
\text { deste estudo. } \\
\text { Com base no } \\
\text { índice de } \\
\text { massa } \\
\text { corporal, } 27 \\
\text { eram } \\
\text { eutróficos, } 10 \\
\text { estavam } \\
\text { acima do peso } \\
\text { e } 25 \text { estavam } \\
\text { obesos. } \\
\text { Os níveis de } \\
\text { triglicerídeos, } \\
\text { colesterol } \\
\text { LDL, HOMA- } \\
\text { RI e leptina } \\
\text { estavam mais } \\
\text { elevados nas } \\
\text { crianças }\end{array}$ & $\begin{array}{l}\text { Este estudo mostrou } \\
\text { que a disfunção } \\
\text { endotelial já está } \\
\text { presente nas crianças } \\
\text { obesas, sugerindo } \\
\text { uma predisposição à } \\
\text { doença } \\
\text { aterosclerótica. Além } \\
\text { disso, os níveis de } \\
\text { colesterol HDL foram } \\
\text { correlacionados à } \\
\text { aptidão física, } \\
\text { independentemente } \\
\text { do índice de massa } \\
\text { corporal. }\end{array}$ \\
\hline
\end{tabular}




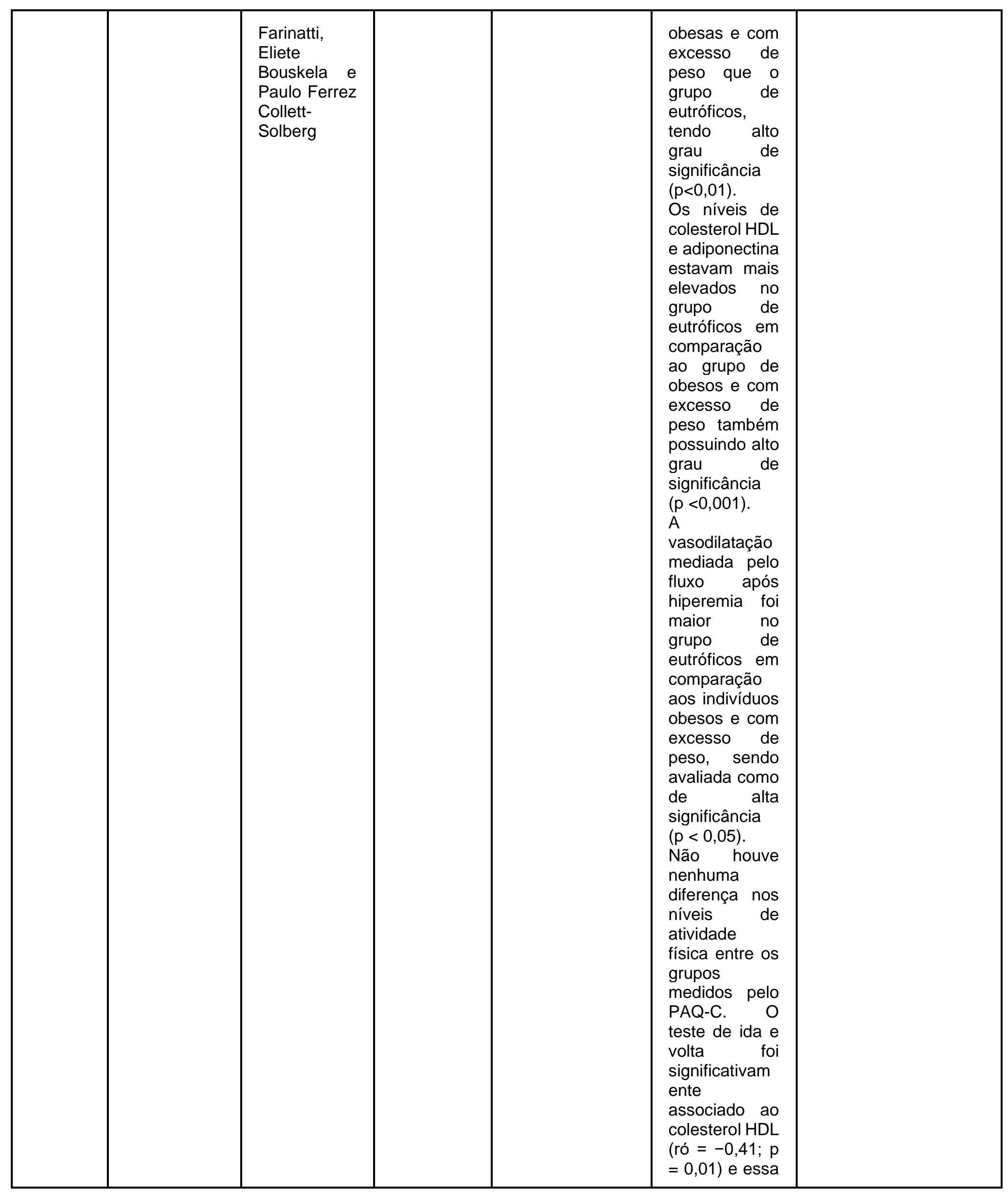




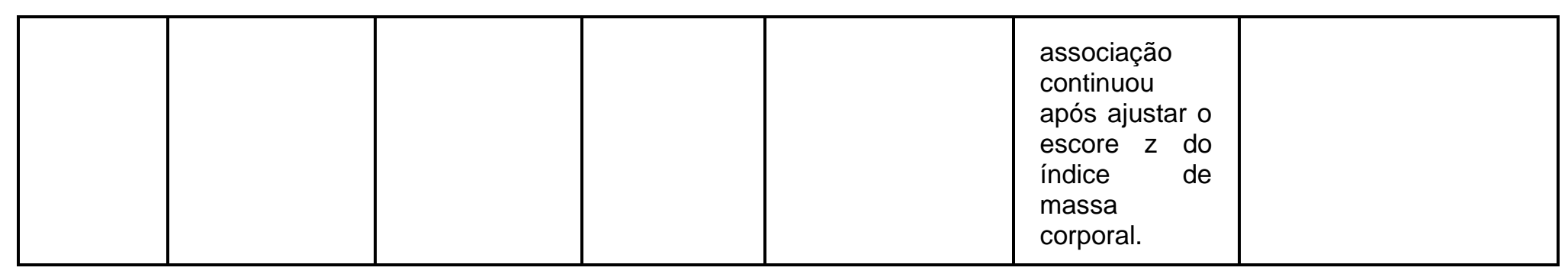

